\title{
DETECTION TECHNIQUES OF CIRCULAR RNAS AS NEW BIOMARKERS IN SYSTEMIC LUPUS ERYTHEMATOSUS
}

\author{
Galya Mihaylova $^{1}$, Mariya Kosturkova ${ }^{2,3}$, Maria Radanova ${ }^{1,4}$ \\ ${ }^{1}$ Department of Biochemistry, Molecular Medicine and Nutrigenomics, \\ Faculty of Pharmacy, Medical University of Varna, Bulgaria \\ ${ }^{2}$ Department of Propaedeutics of Internal Diseases, Faculty of Medicine, \\ Medical University of Varna, Bulgaria \\ ${ }^{3}$ Clinic of Internal Disease, St. Marina University Hospital, Varna, Bulgaria \\ ${ }^{4}$ Clinic of General and Clinical Pathology, Laboratory of Molecular Pathology, \\ St. Marina University Hospital, Varna, Bulgaria
}

\begin{abstract}
Systemic lupus erythematosus (SLE) is severe, chronic autoimmune disease affecting mainly young active individuals, leading to disability and premature death. Recent studies have reported long non-coding RNAs (IncRNAs) to participate in the pathogenesis of the disease. Among lncRNAs, the circular RNAs (circRNAs) gain growing scientific attention due to their stability in body fluids. This makes them suitable for new nonbiomarkers for evaluation of SLE activity and promising therapeutic targets. Methods for detecting of circRNAs are evolving rapidly. The aim of this review is to present these techniques and their advantages and disadvantages.
\end{abstract}

Keywords: $\operatorname{circRNAs,~long~non-coding~RNA,~SLE~}$

\section{INTRODUCTION}

Systemic lupus erythematosus (SLE) is the prototype multisystemic autoimmune disease with a chronic course of remissions and exacerbations, leading in many cases to severe end-organ damage and substantial disability $(1,2)$. Its etiology is complex and includes environmental, hormonal, genetic and epigenetic factors (3). Typical for the disease

Address for correspondence:

Maria Radanova

Faculty of Pharmacy

Medical University of Varna

84 Tzar Osvoboditel Blvd

9002 Varna

Bulgaria

e-mail: maria.radanova@gmail.com

Received: November 24, 2020

Accepted: December 9, 2020 are vigorous autoantibody production, generation of immune complexes and immune-mediated tissue injury $(2,4)$. The clinical presentation can vary among patients from mild mucocutaneous or joint manifestations to life-threatening renal or central nervous system involvement.

Given this heterogeneity, SLE is a challenge not only to treat, but also to diagnose and monitor. That is why in recent years there has been an intensive search of biomarkers for early diagnosis and for more accurate evaluation of disease activity. In the wide spectrum of biomarkers, non-coding RNAs (ncRNAs) appear to have the potential of being the ideal biomarker - specific, sensitive and noninvasive. ncRNAs are the dominant product of eukaryotic transcription and are associated with key biological processes and diseases. Generally, there are: 1) small non-coding RNAs (sncRNAs) with a length equal to 
or less than 200 nucleotides and 2) long non-coding RNAs RNAs (lncRNAs) - with a length of over 200 nucleotides (5). IncRNAs are involved in various important biological processes such as chromatin remodelling, gene transcription, RNA splicing and others. The functional consequences of a change in the expression of lncRNAs are large and pathogenetically significant. Recent studies have shown that lncRNAs are involved in the pathogenesis of immunemediated inflammatory diseases such as rheumatoid arthritis, autoimmune thyroiditis and SLE (6-8). This makes them suitable targets for therapy and allows them to be studied as potential biomarkers for early diagnosis and assessment of activity in SLE (912). Recently, a newly discovered type lncRNAs - circular RNAs (circRNAs) have received great scientific attention. They are resistant to exonucleases and have a stable structure, which prolongs their half-life; they also have a tissue-specific expression and a relation to developmental stage or age abundance (13). Since a growing number of studies have revealed circRNAs as a potential clinical biomarker for SLE (14$17)$ and for lupus nephritis (LN) (11, 18-20), their detection is of great importance. Along with the increasing knowledge about these RNAs, the methods for their detection are also being developed. The aim of this article is to review these techniques and present their advantages and disadvantages.

\section{METHODS FOR CIRCRNAS DETECTION}

In the early studies, the detection of circRNAs has been achieved with electron microscopy (21). Nowadays, various techniques are used to detect circRNA expression and to investigate their functions. For example, by using RNA-Seq and microarray researchers can identify circRNAs (22). Validation methods include polymerase chain reaction (PCR) (23), northern blot (24), in situ hybridization (25). Usage of enzymatic methods like RNase R and RNase $\mathrm{H}$ degradation assay is optional and helps researchers to achieve correct identification of circRNAs $(26,27)$. Distinguishing between circRNAs and linear transcripts is possible with 2D gel electrophoresis (28) and gel trap electrophoresis (29). In circRNA analysis, several methods are combined for detection and validation of this type of lncRNAs. In this context, the order of the used detection methods often is as it follows: circRNA selection, RT PCR, Sanger sequencing (Fig. 1).

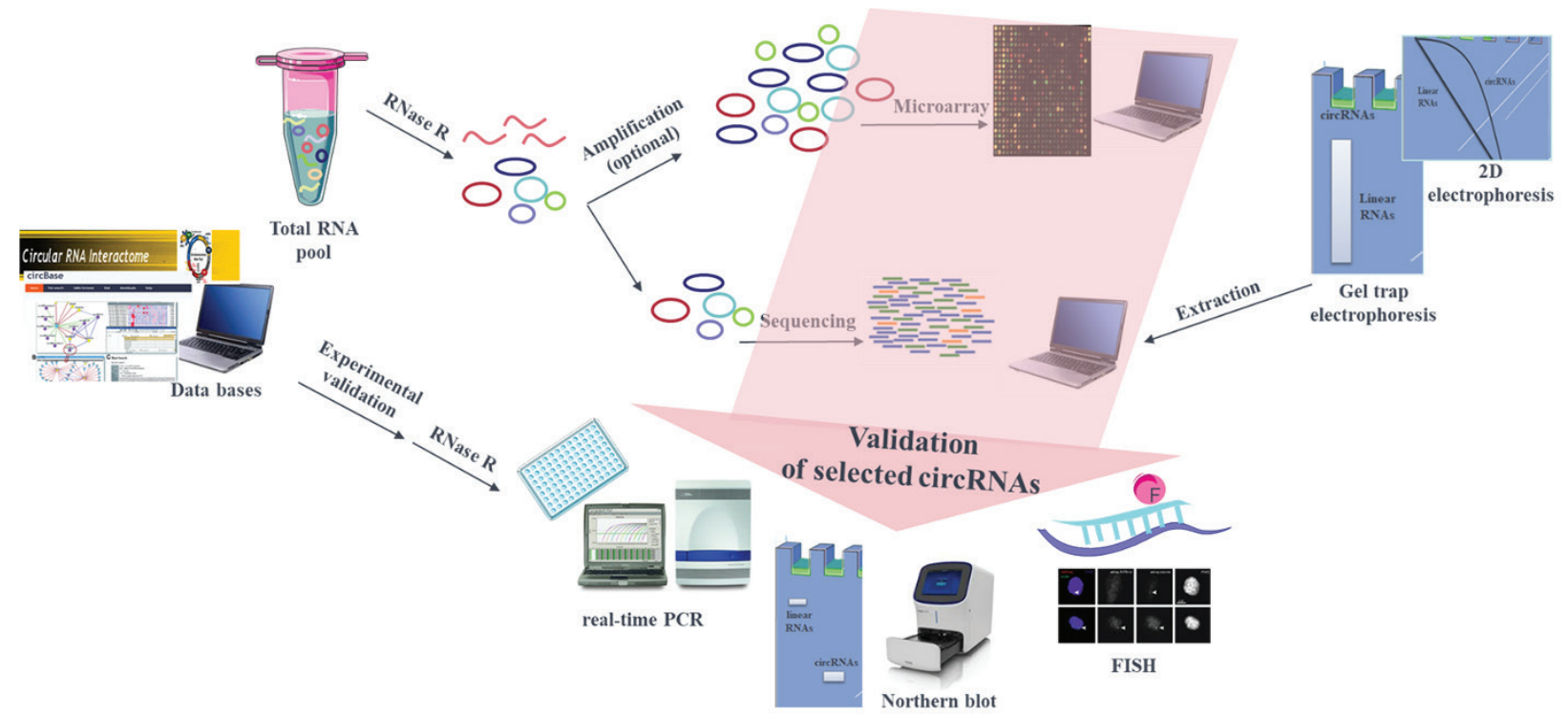

Fig. 1. Workflows in circRNA analysis where different methods for detection and validation are combined. Total pool of RNA can be treated with RNase R and enriched with circRNAs for microarray or sequencing and subsequently data analysis. The amplification step is recommended when there is a low-input material. Next, for validation of circRNAs of interest real time PCR, northern blot or fluorescent in situ hybridization (FISH) can be used. Another strategy begins with electrophoresis - the separated circRNAs are then extracted, sequenced and validated. A third possible workflow starts with circRNA selection from available databases. They give an information about the circRNA sequence, network interactions, expression and disease-related data. PCR technique is the most frequently used one for validation of in silico-selected circRNAs. 
Sylvia Atanasova-Stamova, Svetlana Georgieva, Maya Georgieva

\section{CircRNA Selection}

CircRNAs can be selected from databases, microarray or RNA sequencing. High-throughput analyses like microarray and RNA sequencing include prior treatment with RNase $\mathrm{R}$ for linear RNA reduction and enhancement of circRNA detection (30). If RNA sequencing is performed at high depth (100-150 million reads), enrichment of circRNAs is not necessary (31). Microarrays profile only known circRNAs through hybridization. While microarrays recognize circRNAs based only on the junction sequences, they do give information on the internal sequence of a given circRNA. Moreover, microarray cannot distinguish among circRNAs with common junction sequences (31). However, microarray offers good annotation and analysis related to circRNA biology like miRNA binding sites (32). On the other hand, RNA Seq has limited annotation information and generates large data (32). At the same time, RNA sequencing has a higher discovery power and is recommended for the detection of novel circRNA transcripts (30) (Table 1). For circRNA detection from RNA-Seq data different bioinformatics tools are used. They can be divided into two groups: 1) with "pseudoreferencebased" or "candidate-based" strategy, when they require circRNA sequence with the gene annotation and 2) with "segmented-based" strategy, when they relay backsplicing junction identification from the mapping information generated by alignment of the reads to the reference genome or transcriptome (33). All computational approaches have their own advantages and some authors find dramatic differences in their sensitivity (34). As a conclusion, computational circRNA analyses are still being developed - still the choice of algorithm for the genome-wide detec- tion of circRNA significantly impacts false positive and false negative rates of detection, and they need further development.

Many databases have been developed to facilitate circRNA studies. Some of them provide the sequences of given circRNAs or clarify circRNA position in the genome and expression pattern in tissues and cells (circBase); and propose a design of divergent primers and prediction of miRNA or protein targets (CircInteractome) (33).

\section{Enzymatic Methods}

Enzymatic methods are optional and often performed in combination with other techniques since they provide circRNAs enrichment due to the enzyme-based depletion of linear RNA fragments $(35,36,37)$. Limitations in exoribonuclease RNase R usage are related to incomplete efficiency of the enzyme and the possibility to introduce technical noise $(37,38)$. It is possible that stable structures in linear RNAs block the enzyme action too.

Other less frequent enzymatic methods, which leave circRNAs but not most of the linear RNAs intact, include RNase H (26), tobacco acid phosphatase, and terminator exonuclease treatment (35). When two short DNA probes are annealed to the RNA of interest, RNase H cleaves that RNA. Run on a gel and blotted for the fragments, the circular RNA of interest appears as two bands. If RNA is cleaved in the presence of each DNA probe separately, circular species are shown by one band (38).

Usually, the enzymatic methods go before RNAseq, microarray or RT-PCR analyses in order for true circRNAs to be detected and quantified. However, some linear RNAs such as snRNA can overcome the enrichment step and affect downstream analyses

Table 1. Comparison between microarray and RNA-seq in circRNAs detection

\begin{tabular}{|l|l|}
\hline Microarray & Detect novel circRNAs \\
\hline Detect only known circRNAs & Principle of cloning \& sequencing \\
\hline Principle of hybridization & Low input of total RNA \\
\hline High input of total RNA & May include linear RNAs resistant to RNase R \\
\hline Limited to the probes on the array & Does not inform on variants \\
\hline $\begin{array}{l}\text { Relies on junction sequences and does not inform on } \\
\text { mature sequences }\end{array}$ & Still evolving annotation \\
\hline Good annotation & Large data and high demand of computing power \\
\hline Desktop computational resources & \\
\hline
\end{tabular}


(37). To resolve this problem, Pandey et al. developed the so-called RPAD method, based on initial RNase $\mathrm{R}$ treatment followed by poly(A) tailing and poly(A) depletion (39).

\section{PCR}

The PCR technique is the speediest and most effortless detection method, which is usually used for validation of the selected by microarray or NGS circRNAs. Divergent and not convergent primer pairs are required for expression validation of the selected circRNAs (40). In comparison with convergent primers, divergent ones can amplify the circular form and are resistant to RNase R (41). There are already web tools for the design of divergent primers (42). Linear RNAs, such as GAPDH and $\beta$-actin, are usually used as internal control genes $(43,44)$. There are several studies that have reported that circRNAs are better choice reference genes for the detection of other circRNAs (45). Zhong et al. suggest hsa_circ_0000284 and hsa_circ_0000471 may be used as reference genes for circRNAs and for other RNAs, too (46).

\section{Sanger Sequencing}

When a not characterized or not yet validated circRNA is being analysed, Sanger sequencing of the obtained PCR product is recommended for final validation (40). Sanger sequencing requires purification of PCR products using phenol/chloroform/isoamyl alcohol precipitation, and then the existence of the back-splice site is validated.

\section{Northern Blotting}

In general, the Northern blot starts with denaturation and separation of RNA samples by gel electrophoresis. Agarose gels are recommended for circRNAs from 0.2 up to a few $\mathrm{kb}$. However, if circular and linear RNAs have the same size, they cannot be distinguished by their running behavior. In polyacrylamide gels circRNAs have mobility relative to linear markers, therefore these gels are suitable for circRNAs only up to $1 \mathrm{~kb}$ (24). The separated RNA samples with different size are transferred and immobilized onto a membrane. Then a labelled probe against specific RNA sequences is hybridized with the membrane. After membrane washing and depending on the probe labelling, the hybrid signals are detected through different methods. The specific circRNA detection is determined: 1) by the choice of probe regions and the detection principle; 2) by the choice of a gel matrix (24). So, the design of the probe is one of the important steps in this analysis. The probe usually has 100-200 nt in length and could be selected against continuous exon region or against exon-exon junction. The exonic probe allows simultaneous detection of a linear transcript, thus their usage requires further validation of the circularity by RNase R, for example.

\section{FISH}

Visualization of different RNA species within the cells is allowed by fluorescence in situ hybridization (FISH) (47). When FISH is coupled with highresolution microscopy it becomes a powerful method for analyzing the subcellular localization of RNA. However, the detection of circular RNAs (circRNAs) using microscopy is challenging since circRNA junction is the only target for the FISH probe in order to avoid linear RNA recognition (48).

\section{D Electrophoresis and Gel Trap Electrophoresis}

For distinguishing circRNAs from linear RNAs two-dimensional denaturing polyacrylamide gel electrophoresis and gel trap electrophoresis can also be used. When total RNA is run on a $2 \mathrm{D}$ gel, circular and linear RNAs show different migratory patterns. Linear RNA migration is along the diagonal, whereas circRNA migration is off of the diagonal and resembles an arc (28). circRNAs can be excised from the gel and used in next generation sequencing (NGS) as an enrichment step (49). In gel trap electrophoresis only linear RNAs migrate in the gel. CircRNAs are held in the well, thus forming the circRNA pool. It is possible for circRNAs to be extracted from the gel and sequenced via NGS (49).

\section{CONCLUSION}

The functions of the majority of circRNAs are still unknown. However, circRNA detection and validation are an initial step before biogenesis and functional analysis. That is why the developing of these identification methods will give a precise way to explore the functional relevance of circRNAs that are involved in SLE pathogenesis as well as the opportunity for these circRNAs to be used as biomarkers of a new generation. 
Sylvia Atanasova-Stamova, Svetlana Georgieva, Maya Georgieva

\section{Funding}

This work was supported by the Science Fund of the Medical University of Varna, Bulgaria (Grant number FS-14/11.02.2019)

\section{REFERENCES}

1. Bertsias GK, Pamfil C, Fanouriakis A, Boumpas DT. Diagnostic criteria for systemic lupus erythematosus: has the time come? Nat Rev Rheumatol. 2013;9(11):687-94. doi: 10.1038/nrrheum.2013.103.

2. Rahman A, Isenberg DA. Systemic lupus erythematosus. N Engl J Med. 2008;358(9):929-39. doi: 10.1056/NEJMra071297.

3. D'Cruz DP, Khamashta MA, Hughes GR. Systemic lupus erythematosus. Lancet. 2007;369(9561):58796. doi: 10.1016/S0140-6736(07)60279-7.

4. Tsokos GC. Systemic lupus erythematosus. N Engl J Med. 2011;365(22):2110-21. doi: 10.1056/ NEJMra1100359.

5. Kazimierczyk M, Kasprowicz MK, Kasprzyk ME, Wrzesinski J. Human long noncoding RNA interactome: Detection, characterization and function. Int J Mol Sci. 2020;21(3):1027. doi: 10.3390/ ijms21031027.

6. Wang J, Yan S, Yang J, Lu H, Xu D, Wang Z. Noncoding RNAs in rheumatoid arthritis: From bench to bedside. Front Immunol. 2020;10:3129. doi: 10.3389/fimmu.2019.03129.

7. Taheri M, Eghtedarian R, Dinger ME, GhafouriFard S. Dysregulation of non-coding RNAs in autoimmune thyroid disease. Exp Mol Pathol. 2020;117:104527. doi: 10.1016/j.yexmp.2020.104527.

8. Gao Y, Li S, Zhang Z, Yu X, Zheng J. The role of long non-coding RNAs in the pathogenesis of RA, SLE, and SS. Front Med (Lausanne). 2018;5:193. doi: 10.3389/fmed.2018.00193.

9. Zhang F, Wu L, Qian J, Qu B, Xia S, La T, et al. Identification of the long noncoding RNA NEAT1 as a novel inflammatory regulator acting through MAPK pathway in human lupus. J Autoimmun. 2016;75:96-104. doi: 10.1016/j.jaut.2016.07.012.

10. Suo QF, Sheng J, Qiang FY, Tang ZS, Yang YY. Association of long non-coding RNA GAS5 and miR21 levels in $\mathrm{CD}^{+}{ }^{+} \mathrm{T}$ cells with clinical features of systemic lupus erythematosus. Exp Ther Med. 2018;15(1):345-50. doi: 10.3892/etm.2017.5429.

11. Mihaylova G, Vasilev V, Kosturkova MB, Stoyanov GS, Radanova M. Long non-coding RNAs as new biomarkers in lupus nephritis: A con- nection between present and future. Cureus. 2020;12(7):e9003. doi: 10.7759/cureus.9003.

12. Wu GC, Hu Y, Guan SY, Ye DQ, Pan HF. Differential plasma expression profiles of long non-coding RNAs reveal potential biomarkers for systemic lupus erythematosus. Biomolecules. 2019;9(6):206. doi: 10.3390/biom9060206.

13. Meng $\mathrm{S}$, Zhou H, Feng Z, Xu Z, Tang Y, Li P, Wu M. CircRNA: functions and properties of a novel potential biomarker for cancer. Mol Cancer. 2017;16(1):94. doi: 10.1186/s12943-017-0663-2.

14. Li LJ, Zhu ZW, Zhao W, Tao SS, Li BZ, Xu SZ, et al. Circular RNA expression profile and potential function of hsa_circ_0045272 in systemic lupus erythematosus. Immunology. 2018;155(1):137-49. doi: 10.1111/imm.12940.

15. Li H, Li K, Lai W, Li X, Wang H, Yang J, et al. Comprehensive circular RNA profiles in plasma reveals that circular RNAs can be used as novel biomarkers for systemic lupus erythematosus. Clin Chim Acta. 2018;480:17-25. doi: 10.1016/j.cca.2018.01.026.

16. Zhang C, Wang $X$, Chen $Y, W u ~ Z$, Zhang C, Shi W. The down-regulation of hsa_circ_0012919, the sponge for miR-125a-3p, contributes to DNA methylation of CD11a and CD70 in CD4 $4^{+} \mathrm{T}$ cells of systemic lupus erythematous. Clin Sci (Lond). 2018;132(21):2285-98. doi: 10.1042/CS20180403.

17. Guo G, Wang H, Ye L, Shi X, Yan K, Lin K, et al. Hsa_circ_0000479 as a novel diagnostic biomarker of systemic lupus erythematosus. Front Immunol. 2019;10:2281. doi: 10.3389/fimmu.2019.02281.

18. Ouyang Q, Huang Q, Jiang Z, Zhao J, Shi GP, Yang M. Using plasma circRNA_002453 as a novel biomarker in the diagnosis of lupus nephritis. Mol Immunol. 2018;101:531-8. doi: 10.1016/j. molimm.2018.07.029.

19. Zhou Z, Sun B, Huang S, Zhao L. Roles of circular RNAs in immune regulation and autoimmune diseases. Cell Death Dis. 2019;10(7):503. doi: 10.1038/ s41419-019-1744-5.

20. Cortes R, Forner MJ. Circular RNAS: novel biomarkers of disease activity in systemic lupus erythematosus? Clin Sci (Lond). 2019;133(9):1049-52. doi: 10.1042/CS20180826.

21. Kos A, Dijkema R, Arnberg AC, van der Meide PH, Schellekens H. The hepatitis delta (delta) virus possesses a circular RNA. Nature. 1986;323(6088):55860. doi: $10.1038 / 323558 \mathrm{a} 0$. 
22. Zhao CH, Qu L, Zhang H, Qu R. Identification of breast cancer-related circRNAs by analysis of microarray and RNA-sequencing data: An observational study. Medicine (Baltimore). 2019;98(46):e18042. doi: 10.1097/ MD.0000000000018042.

23. Hansen TB, Wiklund ED, Bramsen JB, Villadsen SB, Statham AL, Clark SJ, et al. miRNA-dependent gene silencing involving Ago2-mediated cleavage of a circular antisense RNA. EMBO J. 2011;30(21):4414-22. doi: 10.1038/emboj.2011.359.

24. Schneider T, Schreiner S, Preußer C, Bindereif A, Rossbach O. Northern blot analysis of circular RNAs. Methods Mol Biol. 2018;1724:119-33. doi: 10.1007/978-1-4939-7562-4_10.

25. You X, Vlatkovic I, Babic A, Will T, Epstein I, Tushev G, et al. Neural circular RNAs are derived from synaptic genes and regulated by development and plasticity. Nat Neurosci. 2015;18(4):603-10. oi: 10.1038/nn.3975.

26. Capel B, Swain A, Nicolis S, Hacker A, Walter M, Koopman P, Goodfellow P, Lovell-Badge R. Circular transcripts of the testis-determining gene Sry in adult mouse testis. Cell. 1993;73(5):1019-30. doi: 10.1016/0092-8674(93)90279-y.

27. Jeck WR, Sharpless NE. Detecting and characterizing circular RNAs. Nat Biotechnol. 2014;32(5):45361. doi: 10.1038/nbt.2890.

28. Tabak HF, Van der Horst G, Smit J, Winter AJ, Mul Y, Groot Koerkamp MJ. Discrimination between RNA circles, interlocked RNA circles and lariats using two-dimensional polyacrylamide gel electrophoresis. Nucleic Acids Res. 1988;16(14A):6597-605. doi: 10.1093/nar/16.14.6597.

29. Schindler CW, Krolewski JJ, Rush MG. Selective trapping of circular double-stranded DNA molecules in solidifying agarose. Plasmid. 1982;7(3):26370. doi: 10.1016/0147-619x(82)90007-5.

30. López-Jiménez E, Rojas AM, Andrés-León E. RNA sequencing and prediction tools for circular RNAs analysis. Adv Exp Med Biol. 2018;1087:17-33. doi: 10.1007/978-981-13-1426-1_2

31. Pandey PR, Munk R, Kundu G, De S, Abdelmohsen K, Gorospe M. Methods for analysis of circular RNAs. Wiley Interdiscip Rev RNA. 2020;11(1):e1566. doi: 10.1002/wrna.1566.

32. Arraystar Inc. Why use microarray over RNA-seq for circular RNA expression profiling? Available from: https://www.arraystar.com/reviews/why-use- microarray-over-rna-seq-for-circular-rna-expression-profiling/

33. Zucko D, Boris-Lawrie K. Circular RNAs are regulators of diverse animal transcriptomes: One health perspective. Front Genet. 2020;11:999. doi: 10.3389/ fgene.2020.00999.

34. Hansen TB, Venø MT, Damgaard CK, Kjems J. Comparison of circular RNA prediction tools. Nucleic Acids Res. 2016;44(6):e58. doi: 10.1093/nar/ gkv1458.

35. Jeck WR, Sharpless NE. Detecting and characterizing circular RNAs. Nat Biotechnol. 2014;32(5):45361. doi: 10.1038/nbt.2890.

36. Suzuki H, Zuo Y, Wang J, Zhang MQ, Malhotra A, Mayeda A. Characterization of RNase R-digested cellular RNA source that consists of lariat and circular RNAs from pre-mRNA splicing. Nucleic Acids Res. 2006;34(8):e63. doi: 10.1093/nar/gkl151.

37. Vincent HA, Deutscher MP. Substrate recognition and catalysis by the exoribonuclease RNase R. J Biol Chem. 2006;281(40):29769-75. doi: 10.1074/jbc. M606744200.

38. Barrett SP, Salzman J. Circular RNAs: analysis, expression and potential functions. Development. 2016;143(11):1838-47. doi: 10.1242/dev.128074.

39. Pandey PR, Rout PK, Das A, Gorospe M, Panda AC. RPAD (RNase R treatment, polyadenylation, and poly(A)+ RNA depletion) method to isolate highly pure circular RNA. Methods. 2019;155:41-8. doi: 10.1016/j.ymeth.2018.10.022.

40. Heumüller AW, Boeckel JN. Characterization and validation of circular RNA and their host gene mRNA expression using PCR. Methods Mol Biol. 2018;1724:57-67. doi: 10.1007/978-1-4939-7562-4_5.

41. Lu T, Cui L, Zhou Y, Zhu C, Fan D, Gong H, et al. Transcriptome-wide investigation of circular RNAs in rice. RNA. 2015;21(12):2076-87. doi: 10.1261/ rna.052282.115.

42. Dudekula DB, Panda AC, Grammatikakis I, De S, Abdelmohsen K, Gorospe M. CircInteractome: A web tool for exploring circular RNAs and their interacting proteins and $\mathrm{mi}-$ croRNAs. RNA Biol. 2016;13(1):34-42. doi: 10.1080/15476286.2015.1128065.

43. Li P, Chen H, Chen S, Mo X, Li T, Xiao B, et al. Circular RNA 0000096 affects cell growth and migration in gastric cancer. Br J Cancer. 2017;116(5):62633. doi: 10.1038/bjc.2016.451. 
44. Zhong $\mathrm{S}$, Wang J, Hou J, Zhang Q, Xu H, Hu J, et al. Circular RNA hsa_circ_0000993 inhibits metastasis of gastric cancer cells. Epigenomics. 2018;10(10):1301-13. doi: 10.2217/epi-2017-0173.

45. Tu C, Du T, Shao C, Liu Z, Li L, Shen Y. Evaluating the potential of housekeeping genes, rRNAs, snRNAs, microRNAs and circRNAs as reference genes for the estimation of PMI. Forensic Sci Med Pathol. 2018;14(2):194-201. doi: 10.1007/ s12024-018-9973-y.

46. Zhong S, Zhou S, Yang S, Yu X, Xu H, Wang J, et al. Identification of internal control genes for circular RNAs. Biotechnol Lett. 2019;41(10):1111-9. doi: 10.1007/s10529-019-02723-0.
47. Itzkovitz $S$, van Oudenaarden A. Validating transcripts with probes and imaging technology. Nat Methods. 2011;8(4 Suppl):S12-9. doi: 10.1038/ nmeth.1573.

48. Zirkel A, Papantonis A. Detecting circular RNAs by RNA fluorescence in situ hybridization. Methods Mol Biol. 2018;1724:69-75. doi: 10.1007/978-1-4939-7562-4_6.

49. Awan AR, Manfredo A, Pleiss JA. Lariat sequencing in a unicellular yeast identifies regulated alternative splicing of exons that are evolutionarily conserved with humans. Proc Natl Acad Sci U S A. 2013;110(31):12762-7. doi: 10.1073/pnas.1218353110. 